\title{
Impact of Random Waypoint Mobility on Reactive Routing Protocols of Scalable Mobile Ad hoc Network
}

\author{
Poonam Mittal ${ }^{1}$, Sneha Kanchan ${ }^{2}$ \\ ${ }^{I}$ (Assistant Professor Computer Science, YMCA University, India) \\ ${ }^{2}$ (Research Scholar Computer Science, YMCA University, India)
}

\begin{abstract}
Mobile Ad hoc networks are the self-configuring network, in which there is no centralized access point. The mobile nodes itself act as routers for one another. MANET is basically a wireless ad hoc network with the routing capability. Scalability and Mobility have measure impact on the behavior of ad hoc routing protocols. Before deploying these protocols on the network, we must observe their performance under various circumstances. Simulation is a very effective and efficient technique for measuring their behavior in this decentralized infrastructure less network. This paper focuses on the characteristic measure of reactive routing protocols (DSR, AODV, DYMO, IERP and LAR1) for different criteria on application layer and transport layer of Internet . We compare the behavior of these protocols with the increasing number of nodes using Random Waypoint Mobility Model and taking pause time as the main criterion. Traffic load is constant and network load is varying in this analysis.
\end{abstract}

Keywords: Ad hoc networks, Pause time, QualNet, Random Waypoint mobility, Routing protocols, Simulation

\section{Introduction}

The ad hoc routing protocols are deployed according to the need of communication because these pose different criteria under various circumstances. It is not feasible to deploy the network first with any protocol, and then test it. If the network fails to fulfill the criteria after deployment, it is very costly to redesign it using another protocol. Hence it is a need to compare the protocols' behavior prior to its use and this is done by simulation. In simulation, we have an artificial, but exact environment of the real network. Here, we are comparing the performance of different reactive routing protocols as it is essential to analyze their behavior and effectiveness [1].

In this research, we concentrated mainly on reactive routing protocol. Qualitatively, these protocols are preferred over proactive routing protocols [2]. The main issue is to find various ways to improve the runtime performance of the protocols. In reactive protocols, path is established when needed, which reduces overhead of regular updating of the table. But it reduces the throughput of these protocols. In reactive routing, we do not maintain regular table for several routes from source to destination [3]. The source node sends route request packet (RREQ) to its neighbors and all neighbors again broadcast the message to their neighbors and next again do the same and in this way the route is find out and the reply packet (RREP) is sent back to the source following the same path that of RREQ packet [4].

In the remaining part of this paper, we have described each section in the following manner. The various types of protocols used in our research are given in section 2. In section 3, we describe the mobility models and in section 4, we describe the simulation setup and its environment and next to it, in section 5, we discuss over the expected result of our simulation and describe the actual outcome of it. However, in section 6 , we conclude our research with some future scope of this.

\section{Protocols Specifications} follows:

In our paper, we are discussing about the reactive protocols. The various reactive protocols are as

\subsection{Dynamic Source Routing}

In Dynamic Source Routing (DSR), route is established by flooding the route request messages. Each node maintains a route cache in which the recent routes are being cached [2]. Whenever there is a need of a route, and if the route is in cache, it is returned immediately without the need of moving up to the destination [5]. In this routing, source determines exact sequence of nodes through which packet is propagated. This mechanism of "Route Discovery" and "Route Maintenance" are the major components of DSR [6] [7]. It eliminates the periodic update feature of DSDV and other Proactive routing protocols. DSR allows the senders to select and control the route. 


\subsection{Ad-Hoc On-Demand Distance Vector}

Ad- Hoc On-Demand Distance Vector (AODV) is the protocol which includes the best features of DSR, on demand routing mechanism of route discovery and route maintenance. This feature is combined with hop by hop routing sequencing number and periodic packets updating feature of Destination Sequenced Distance Vector (DSDV) protocol. When a route is demanded and if it is not available, a route request (RREQ) message is generated and flooded in a limited way to its neighbors [7]. Initial delay is caused to transmit the packet to the selected nodes but it controls the traffic. Once the RREQ is reached to its destination or to the node, which has the path up to the route in its cache, the route is found [6]. After establishing the connection, message is sent to the destination. AODV is highly adaptive to the dynamic networks and also are very scalable to the large network [8].

\subsection{Dynamic MANET on-Demand}

Dynamic MANET on-Demand (DYMO) routing protocol is very recently proposed protocol which is basically an AODV protocol but removes unnecessary Route Reply (RREP) packets. It enjoys the "Path accumulation" property of DSR and simplifies AODV by removing the precursor lists and Hello messages. Hence it includes multi-hop routing property of proactive along with the reactive approach. It has two basic operation "route discovery" and "route management". In discovering the route, RREQ is flooded throughout the network to find out the target node. After receiving the RREQ, target responds by sending the RREP packets towards the source. The transmission of packets is in hop-by-hop fashion. When the source receives the RREP, then the connection is established between the source and the target node. While maintaining a route, a Route ERROR packet, RERR, containing the list of unreachable nodes is broadcasted. It indicates that the route is broken. The receiving node checks in its cache that if the listed node is there or not. If yes, the entry is invalidated and if not, the RERR is broadcasted again. By using sequence numbers, DYMO also enjoys the loop free routing.

\subsection{Inter zone Routing Protocol}

Inter zone Routing Protocol (IERP) is the reactive approach of the zone based routing technique of hybrid protocol. In hybrid routing, nodes have predefined path up to some level but after crossing that level, it has to find its route only when demanded. IERP is the protocol responsible for finding the paths which are not within that level means that routing zone. It broadcasts using unicast routing to send the packet to the boundary and then to the peripherals of the current zone. If a route is found by any node, then it replies it. Otherwise the request is transmitted to the further peripheral zones.

\subsection{Location Aided Routing}

Location Aided Routing (LAR1) uses the location information of the mobile nodes. Locations can be categorized into two parts, expected zone and request zone. According to the previous position of the node, some zones are defined as the expected zone of the node. The RREQ is only sent to that expected zone. The zone which includes the expected zone and the surrounding is known as request zone. Hence the RREQ is only flooded in the request zone and so, it reduces very large traffic overhead. Once the destination node receives the RREQ, it replies with its position, speed and the current time.

\section{Mobility Model-Random Waypoint Mobility Model}

For the performance evaluation of a protocol of an ad hoc network, the protocol should be tested under realistic conditions, e.g., the limited buffer space, data traffic models, and realistic movement of mobile users (mobility models). In order to thoroughly simulate a protocol for an ad hoc network, an accurate mobility model is the first requirement. Mobility model is mainly subdivided into three models: File Mobility Model, Random Waypoint Mobility Model and Group Mobility Model [9]. In random waypoint mobility model, nodes move randomly and, are independent of each other. They can pause for few seconds while changing the direction and speed [10]. This interval is fixed for a scenario and known as pause time. After completion of this time, the host moves towards the chosen waypoint at the selected speed [10]. Then again it waits for the constant interval before moving towards new waypoint. This process always continues in the network. Hence, Pause time is the time period for which the nodes in the mobile ad hoc network do not move anywhere. It plays a very important role in route establishment. Suppose, the nodes are always moving; their neighbors will change too frequently to make a session for transmitting the packets. It is also possible that even after making the route, the nodes move out of range of its neighbors, hence breaking the route. Then the source node has to reinitiate the whole routing process again. So, it is a wise decision for the nodes to pause for few seconds to complete their communication. In this paper, we propose the simulation based upon Random Waypoint mobility model with the pause of 10 seconds in a scalable network. 


\section{Simulation Setup and Performance Metrics}

As said in the above paragraphs, we are evaluating the performance of the different reactive routing protocols on ad hoc network. So, main agenda of this simulation is to analyze the behavior of these protocols on ad hoc environment. For this, we have used a very scalable and portable simulation tool, QualNet 5.0 [11]. The terrain used is of $1500 * 1500$ areas. Moreover number of routers varies from 10,20 and $30 \ldots$ up to 120 nodes.

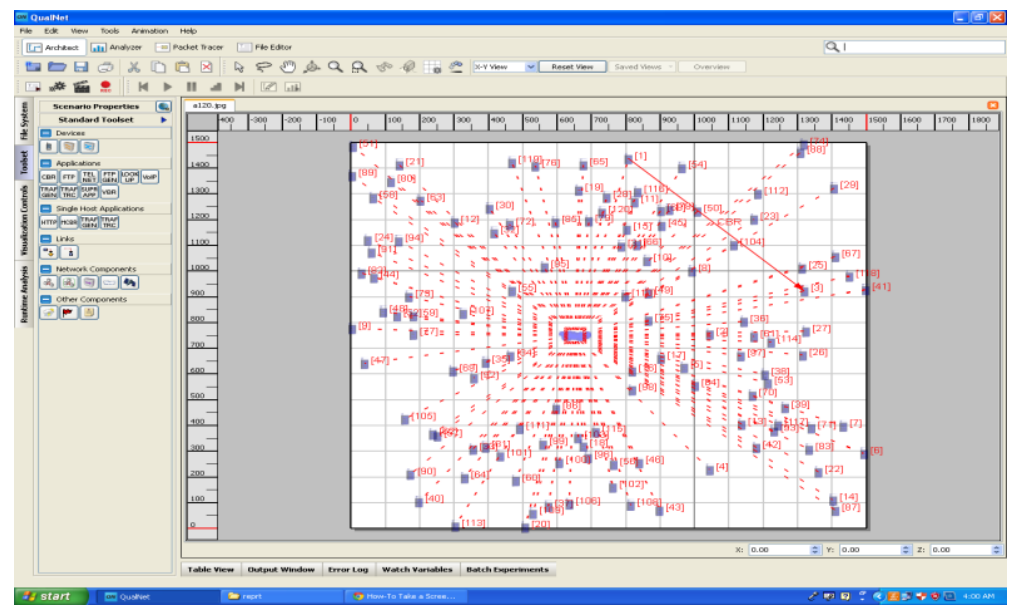

Fig. 1 Design scenario of 120 nodes

The mobility model used is Random Waypoint mobility model and pause time taken is of 10 seconds. The data is sent at constant bit rate with the throughput of 4274 bits per second. Moreover total number of packets sent is 24 and total number of bytes sent is 12288.Energy model used is mica motes and battery model is linear. In Fig. 1, a scenario with 120 nodes is shown in design mode. The nodes were randomly distributed in 1500 X 1500 unit area. In Fig. 2 scenario with 60 nodes is shown in simulation mode. The simulation was run for 30 seconds. The reactive routing protocols taken were AODV, DSR, DYMO, IERP and LAR1.

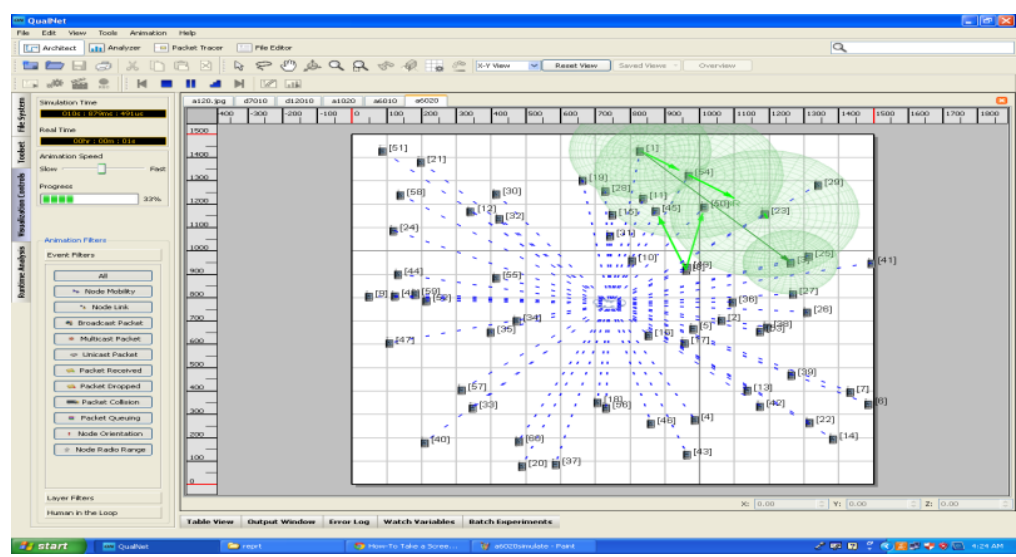

Fig. 2 Design scenario in simulation mode

\section{Results and Discussions}

This section illustrates the result of our research. The parameters' values are investigated by varying the load. Number of iteration for each experiment is 10 . The protocol which posses the best and consistent behavior, that is taken as the best protocol for that parameter. First of all application layer parameters are discussed.

\subsection{Average Jitter}

As shown by the fig. 3, in reactive protocols, DSR has very consistent performance. The delay in between the packets is less and so average jitter is least for this protocol. 


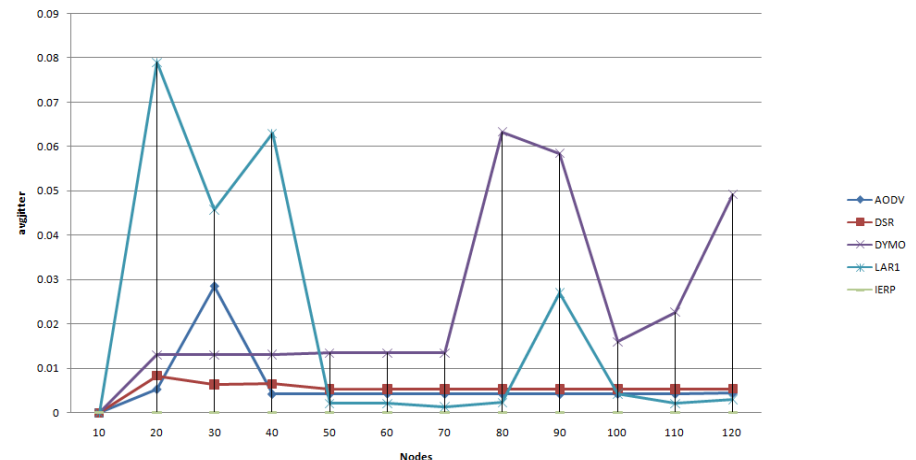

\subsection{First Packet Received}

Fig. 3 Average jitter

Fig. 4 tells that LAR1 is the quickest in receiving the first packet. That means it responds very fast. It has an additional advantage that it uses location information of the nodes. DSR is also very quick and it is consistently receiving the first packet with smaller delay.

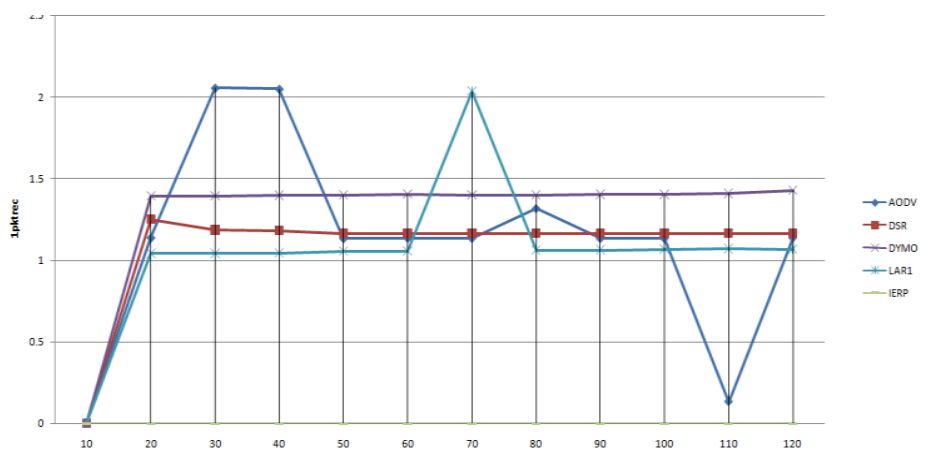

\subsection{Total bytes and packets sent}

Fig. 4 First Packet received

In Fig. 5 and fig. 6, we can see that networks using DSR receives the maximum bytes and packets. Hence the least number of packets are lost in this case. So, it's the most reliable protocol amongst reactive routing protocols. After DSR, AODV is the most reliable protocol. LAR1 is the least reliable one as it loses many packets.

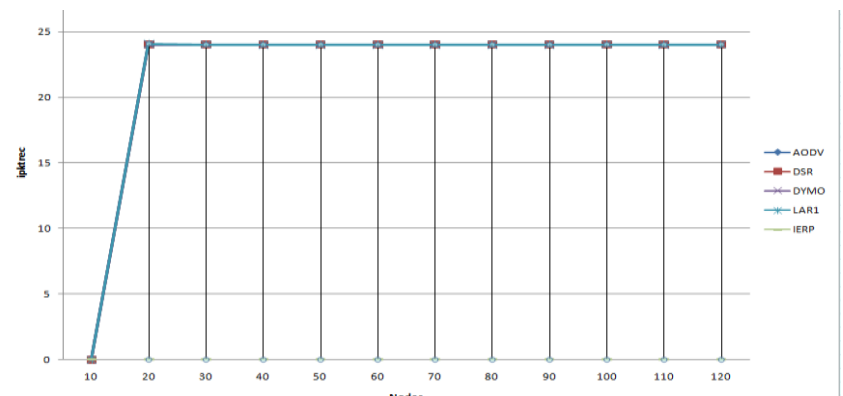

Fig. 5 Total bytes sent

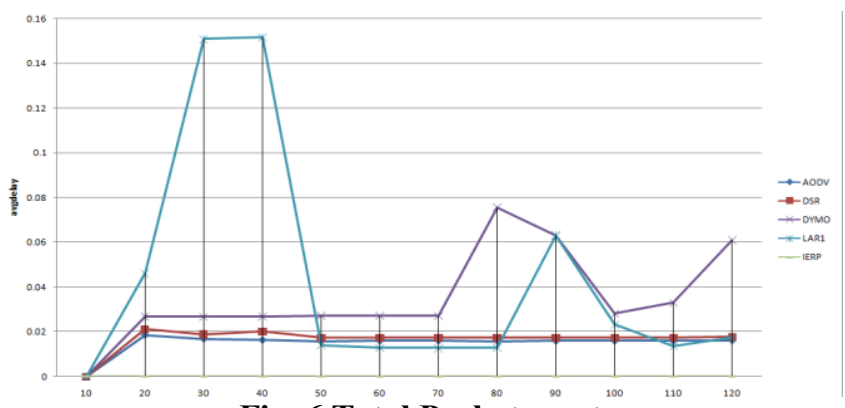

Fig. 6 Total Packets sent 


\subsection{Last Packet Received}

The study shows that last packet is received almost in same time by all the protocols. By investigating closely, DYMO is the fastest protocol among all reactive protocols. This is illustrated in fig.7.

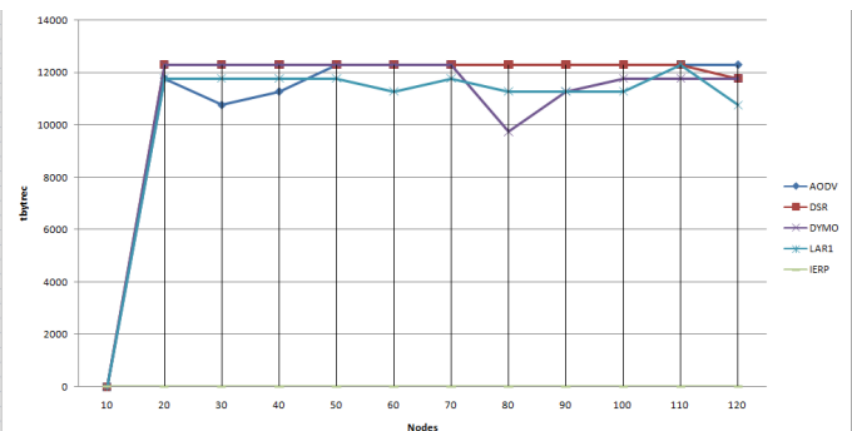

Fig. 7 Last packet received

\subsection{Average end to end delay}

Fig.8 shows that minimum average end to end delay is incurred by AODV in almost all the cases. It is because AODV possess lower setup delay for connections and detection of latest route to the destination.

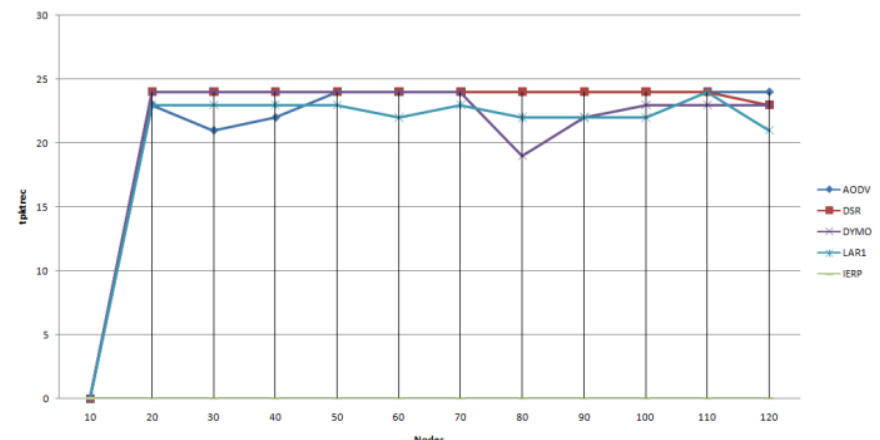

Fig. 8 Average End to End delay

\subsection{Packets from application and Packets to the application}

These come under transport layer criteria. In fig.9, we have a graph of behavior of protocols on the parameter, packet from application. In reactive routing protocol, all applications send same number of packets to the network. But in fig. 10, which is for packet to application, we can see that packets received by the applications are not same. Furthermore discussing about the best among all and that is DSR.

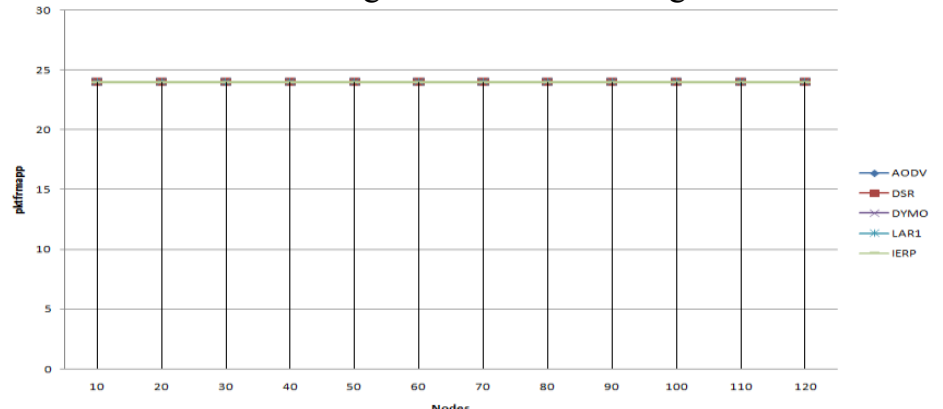

Fig. 9 Packets from application

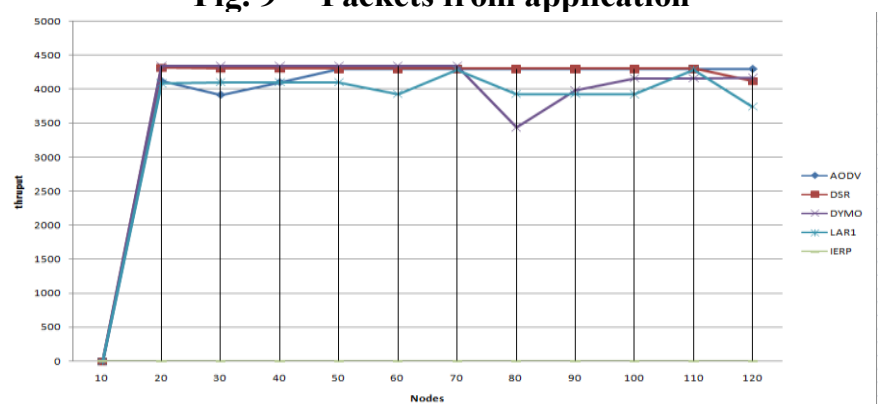

Fig. 10 Packet to application 


\section{Conclusion and future work}

In this paper, we have investigated the reactive routing protocol and analyzed the best protocol for each criterion. Our research finds the best protocol among proactive, reactive and hybrid protocols. DSR is the best protocol and showing consistently good performance. Although, average end to end delay of DSR is higher than AODV, both can send maximum data in minimum time. So these are the most feasible protocol. DYMO is also performing well when the load is less. But as we increase the number of nodes in the network, a decline in the graph of DYMO is clearly visible. In future, this research can be extended to proactive as well as hybrid protocols.

\section{References}

[1] Md. Saiful Azad, Farhat Anwar, Md. Arafatur Rahman, Aisha H. Abdalla, Akhmad Unggul Priantoro and Omer Mahmoud, Performance Comparison of Proactive and Reactive Multicast Routing Protocols over Wireless Mesh Networks, IJCSNS International Journal of Computer Science and Network Security, VOL.9 No.6, June 2009.

[2] C. Sivaram Murthy and B. S. Manoj, Ad Hoc Wireless Networks, Architecture and Protocols (Prentice-Hall PTR, 2004).

[3] Ravinder Ahuja, Simulation based Performance Evaluation and Comparison of Reactive, Proactive and Hybrid Routing Protocols based on Random Waypoint Mobility Model, International Journal of Computer Applications (0975 - 8887)Volume 7No.11, October 2010.

[4] Zhenqiang Ye, Srikanth V. Krishnamurthy and Satish K. Tripathi, A Framework for Reliable Routing in Mobile Ad Hoc Networks,Proceedings of the IEEE INFOCOM, 2003.

[5] Valentina Timcenko, Mirjana Stojanovic and Slavica Bostjancic Rakas, MANET Routing Protocols vs. Mobility Models:Performance Analysis and Comparison, Institute Mihailo Pupin, Volgina 15, 11060 Belgrade, SERBIA.

[6] Changling Liu and Jorg Kaiser, A Survey of Mobile Ad hoc network routing protocols, Department of Computer Structure, University of ULM, Germany, 2003.

[7] Yasser Kamal Hassan, Mohamed Hashim Abd El-Aziz and Ahmed Safwat Abd El-Radi, Performance Evaluation of Mobility Speed over MANET Routing Protocols, International Journal of Network Security, Vol.11, No.3, PP.128\{138, Nov. 2010.

[8] Harminder Qian Feng, Zhongmin Cai, Jin Yang, Xunchao Hu, A Performance Comparison of Ad Hoc Network Protocols, Second International Workshop on Computer Science and Engineering, IEEE, 2009.

[9] S. Bindra, Sunil K. Maakar and A. L. Sangal, Performance Evaluation of Two Reactive Routing Protocols ofMANET using Group Mobility Model, IJCSI International Journal of Computer Science Issues, Vol. 7, Issue 3, No 10, May 2010.

[10] Tracy Camp, Jeff Boleng, Vanessa Davies, A Survey of Mobility Models for Ad Hoc Network Research, Wireless Communication \& Mobile Computing (WCMC) :: Special issue on Mobile Ad Hoc Networking: Research, Trends and Applications, vol. 2, no. 5, pp. 483-502, 2002.

[11] Tobias Doerffel, Simulation of wireless ad-hoc sensor networks with QualNet, Technische Universitat Chemnitz, 2009. 